\title{
A surprise with many-flavor staggered fermions in the strong coupling limit
}

\author{
Philippe de Forcrand \\ Institut für Theoretische Physik, ETH Zürich, CH-8093, Switzerland \\ CERN, Physics Department, TH Unit, CH-1211 Geneva 23, Switzerland \\ E-mail: forcrandephys.ethz.ch
}

\section{Seyong Kim}

Department of Physics, Sejong University, Seoul 143-747, Korea

E-mail: skimesejong.ac.kr

\section{Wolfgang Unger*}

Institut für Theoretische Physik, Goethe-Universität Frankfurt, 60438 Frankfurt am Main, Germany

E-mail: ungereth.physik.uni-frankfurt.de

\begin{abstract}
It is widely believed that chiral symmetry is spontaneously broken at zero temperature in the strong coupling limit of staggered fermions, for any number of colors and flavors. Using Monte Carlo simulations, we show that this conventional wisdom, based on a mean-field analysis, is wrong. For sufficiently many fundamental flavors, chiral symmetry is restored via a bulk, first-order transition. This chirally symmetric phase appears to be analytically connected with the expected conformal window of manyflavor continuum QCD. We perform simulations in the chirally symmetric phase at zero quark mass for various system sizes $L$, and measure the torelon mass and the Dirac spectrum. We find that all observables scale with $L$, which is hence the only infrared length scale. Thus, the strong-coupling chirally restored phase appears as a convenient laboratory to study IR-conformality. Finally, we present a conjecture for the phase diagram of lattice QCD as a function of the bare coupling and the number of quark flavors.
\end{abstract}

The 30 International Symposium on Lattice Field Theory - Lattice 2012,

June 24-29, 2012

Cairns, Australia

\footnotetext{
*Speaker.
} 


\section{Introduction}

The possibility that the Higgs boson could be a composite bound-state in a high-energy Technicolor theory, together with the requirement that such a theory be "walking" in order to accommodate stringent bounds on flavor-changing neutral currents, has motivated several large-scale computer simulations. To determine via Monte Carlo simulations whether a given theory with specific gauge group and fermion content is inside the conformal window, i.e. possesses an infrared fixed point (IRFP), is particularly challenging: one must probe the extreme infrared properties of the theory, while at the same time taking the continuum limit, and controlling the chiral limit. Due to these difficulties, in spite of considerable efforts, there is no consensus yet on the minimum number $N_{f}{ }^{*}$ of fundamental quark flavors needed for QCD to be inside the conformal window [1]. ${ }^{1}$

Here, we relax the demand that results should be obtained in the continuum limit. On a coarse lattice, long-distance properties can be studied more economically. While such properties may differ from those of the corresponding continuum theory, it is still instructive to consider the possible existence of an IRFP for a discretized lattice theory. The phase diagram of $\mathrm{SU}\left(N_{c}\right)$ gauge theory with $N_{f}$ fundamental fermions, as a function of $N_{f}$ and the bare gauge coupling, has been predicted in the celebrated Ref. [3]. It is important to confront these predictions with uncontroversial numerical evidence. Therefore, we start our investigation by considering the strong coupling limit, where the lattice is maximally coarse. ${ }^{2}$ The conventional wisdom for strong coupling QCD with staggered fermions is that chiral symmetry always remains broken at zero temperature, regardless of the values of $N_{f}$ and $N_{c}$. This belief is based on meanfield analyses performed in some of the earliest papers on lattice QCD. In particular, it was shown in [4] that at leading order in a $1 / d$ expansion, the chiral condensate has a value independent of $N_{f}$ and $N_{c}$, and depends only on the number $d$ of spatial dimensions, $\langle\bar{\psi} \psi\rangle=\sqrt{\frac{2}{d}}\left(1-\frac{1}{4 d}\right)$. Chiral symmetry may be restored by increasing the temperature $T,{ }^{3}$ but will never be restored at $T=0$. Since naively mean-field theory is expected to work well when the number of d.o.f. per site is large, there was no reason to doubt this result. Besides, it is in accord with the intuition that the gauge field is maximally disordered in the strong-coupling limit, and the disorder drives chiral symmetry breaking. On the other hand, one might expect the above disorder to be modified by dynamical fermions, which have an ordering effect. Indeed, the loop expansion of the determinant shows that the fermionic effective action $S_{\text {eff }}=-\log \operatorname{det}\left(\not D+m_{q}\right)$ starts with a positive plaquette coupling $\Delta \beta$, proportional to $1 / m_{q}^{4}$ for heavy quarks [6]. Clearly, for $N_{f}$ flavors, $S_{\text {eff }} \propto N_{f}$, and hence $\Delta \beta \propto N_{f}$. This plaquette term suppresses fluctuations in the gauge field, which suggests that chiral symmetry restoration might take place for sufficiently large $N_{f}$.

\section{Monte Carlo results}

The only way to resolve this puzzle is to perform Monte Carlo simulations in the strong coupling limit of staggered fermions, to detect a possible chiral symmetry restoration for sufficiently large $N_{f}$. These simulations are straightforward, using the standard HMC algorithm. As expected, the effect of increasing $N_{f}$ on the chiral condensate is to reduce its magnitude. But it came as a surprise to find that the chiral condensate vanishes via a strong first-order transition at $N_{f}^{c} \simeq 52$ continuum flavors in the chiral limit (i.e. $N_{f}^{c} / 4 \simeq 13$ staggered fermion fields). In the broken phase, $\langle\bar{\psi} \psi\rangle$ remains almost constant. It vanishes in the chiral limit due to finite-size effects only. In contrast, in the chirally restored phase the condensate is caused by explicit symmetry breaking and is proportional to the quark mass. This is

\footnotetext{
${ }^{1}$ A numerical demonstration of walking has been provided in the 2- $d O(3)$ model at vacuum angle $\theta \approx \pi$ [2].

${ }^{2}$ Note that we consider standard staggered fermions, and the standard plaquette action. Other discretizations could lead to different results, only the continuum limit is universal.

${ }^{3}$ We find, following the approach of [5], that $T_{c}$ is non-zero for all $N_{f}: a T_{c}=\frac{d}{4}+\frac{d}{8} \frac{N_{c}}{N_{f}}+\mathscr{O}\left(\frac{1}{N_{f}^{2}}\right)$
} 

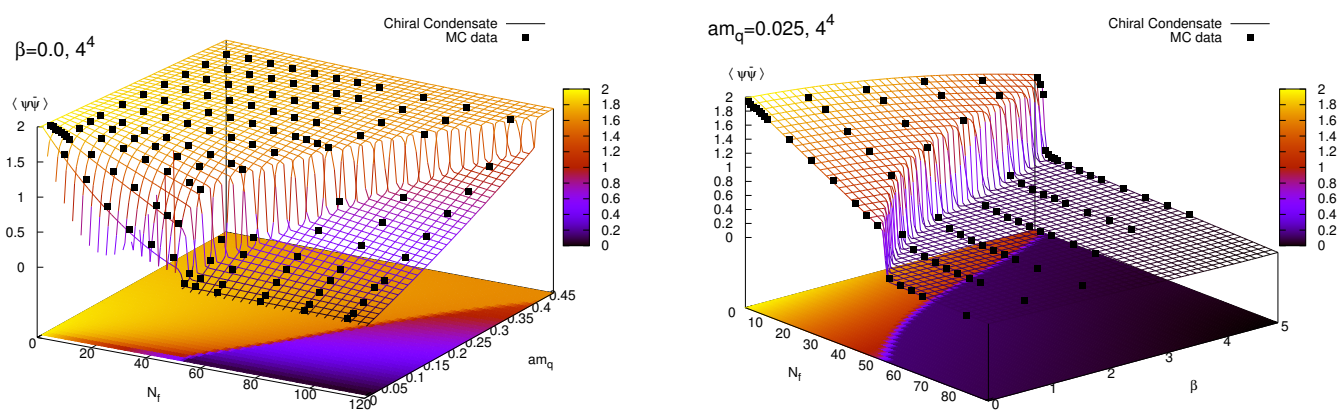

Figure 1: The chiral condensate, left: at strong coupling, $\beta=0$, in the $\left(N_{f}, a m_{q}\right)$ plane, for $4^{4}$. Right: at $a m_{q}=0.025$ in the $\left(N_{f}, \beta\right)$ plane, for $4^{4}$. We have compared with larger volumes [13] and find that the phase transition remains strongly first order at weaker coupling. The contour plot indicates the qualitative behaviour of the phase boundary, extending smoothly to smaller $N_{f}$ at weaker coupling.

illustrated Fig. 1 (left), where the condensate is shown as a function of $N_{f}$ and bare quark mass $\left(a m_{q}\right)$. This $N_{f}$-driven transition turns out to be a bulk, zero-temperature transition: finite-size effects on the phase boundary are small when comparing two different system sizes $4^{4}$ and $6^{4}$. The critical number of flavors increases with the quark mass. ${ }^{4}$

Having established an $N_{f}$-driven phase transition in the strong-coupling limit, we may consider its impact on the lattice theory at non-zero lattice gauge coupling $\beta$ as well. Since the transition is strongly first order, it has to persist for some range in $\beta$ at least. In fact, we find a smooth variation of the $N_{f}$-driven transition with $\beta$ at a given small quark mass $a m_{q}=0.025$, as shown Fig. 1 (right). The transition extends to weak coupling, at least to $\beta=5$, and remains strongly first-order. Thus, it is plausible that this transition, which separates a chirally broken (small $N_{f}$ ) and a chirally symmetric (large $N_{f}$ ) phase, persists all the way to the $\beta \rightarrow \infty$ continuum limit, where it is to be identified with the transition at $N_{f}=N_{f}{ }^{*}$ between the chirally broken and the IR-conformal, chirally symmetric phase. In other words, our chirally restored phase may be analytically connected to the conformal window in the continuum limit, because we do not observe any additional non-analyticity as $\beta$ is increased. This possibility motivates our study of the properties of the strong-coupling chirally symmetric phase, looking for tests of IR-conformality.

\section{Looking for conformality in the chirally symmetric phase}

It is natural to ask whether the chirally restored phase is connected to the conformal window, i.e. whether the chirally restored phase at strong coupling is IR-conformal, and if so, whether it is trivial (IR fixed point coupling $g^{*}=0$ ). In this section we present measurements of gluonic and fermionic observables chosen to address these questions. Our results support the following conclusion: the strongcoupling chirally symmetric phase is IR-conformal, and it is non-trivial. The simulations performed here are all in the chirally symmetric phase at zero plaquette coupling, with $N_{f}=56$ and 96 continuum flavors, and with lattices of size $4^{3} \times 16$ to $12^{3} \times 24$. The quark mass is set exactly to zero, which is computationally feasible because the Dirac operator has a spectral gap in the symmetric phase. Moreover, having one infrared scale, the system size $L$, rather than two scales $\left(L\right.$ and $\left.1 / m_{q}\right)$ is of great advantage when analyzing the results. ${ }^{5}$

\footnotetext{
${ }^{4}$ Heavier quarks have a weaker ordering effect, so that the induced plaquette coupling $\Delta \beta$ decreases if one keeps $N_{f}$ fixed. It takes more flavors to keep the system chirally symmetric: $N_{f}^{c} \propto\left(a m_{q}\right)^{4}$.

${ }^{5}$ Note that the average plaquette values we measure, $\approx 0.35\left(N_{f}=56\right)$ and $\approx 0.52\left(N_{f}=96\right)$, are very far from $0\left(N_{f}=0\right.$,
} 
- The "torelon" is a gluonic excitation which is topologically non-trivial: it is excited by any Wilson line which wraps around the spatial boundary in one spatial direction $i=1,2,3$ : $T_{i}(t)=\operatorname{Tr} \prod_{k=0}^{L-1} U_{i}(\vec{x}+k \hat{i}, t)$. We extract the mass of this excitation from the exponential decay of the correlator $\left\langle T_{i}(0)^{*} T_{i}(t)\right\rangle$. To suppress excited states, we smear the links within each time-slice before constructing $T_{i}$. This observable has been used for a long time to extract the string tension $\sigma$ in Yang-Mills theories [7]: it can be viewed as a loop of gluonic string, whose energy $m_{T}(L)$ grows with its length as $\sigma L$. Our naive expectation that $m_{T}(L)$ grows with $L$ until string breaking occurs was however disproved by our measurements: the dimensionless quantity $\operatorname{am}_{T}(L)$ decreases on larger lattices corresponding to a larger ratio $L / a$. Clearly, our theory is not confining. Moreover, as shown Fig. 2 left, the combination $\operatorname{Lm}_{T}(L)$ is approximately constant as $L$ is increased: the torelon mass varies as $1 / L$. Thus, there is no intrinsic mass scale which appears in this channel: the torelon mass is set by the system size $L$. This remarkable result is our first evidence that our theory is IR-conformal. By relabelling the spatial direction $i$ as the imaginary time direction, one realizes that we are measuring the correlation of two time-like Polyakov loops, whose decay rate is governed by the Debye mass, given perturbatively at lowest order by $m_{D}(T)=2 g T \sqrt{\frac{N_{c}}{3}+\frac{N_{f}}{6}}$. This expression allows us to define a running coupling $g(L)$ via

$$
g(L) \equiv \frac{L m_{T}(L)}{2}\left(\frac{N_{c}}{3}+\frac{N_{f}}{6}\right)^{-1 / 2}
$$

and we see that, in this scheme, our running coupling seems to go to a non-zero constant as $L$ increases (although one cannot exclude, of course, that it slowly goes to zero). Therefore, we have numerical evidence supporting the view that our strong-coupling, chirally symmetric theory is IR-conformal and non-trivial. $^{6}$

- We now turn to the spectrum of the Dirac operator. We have analyzed the Dirac eigenvalue spectrum of the configurations in our Monte Carlo ensembles, using a Lanczos algorithm to obtain an approximation of the whole spectral density and an Arnoldi method to extract the smallest eigenvalues to high accuracy. The observable shown in Fig. 3 is the integrated eigenvalue density, defined as:

$$
v(\lambda)=\int_{0}^{\lambda} \rho(\bar{\lambda}) d \bar{\lambda}=\frac{\operatorname{rank}(\lambda)}{\operatorname{rank}(\operatorname{Dirac} \text { matrix })} \in[0,1],
$$

which counts the fraction of eigenvalues smaller than $\lambda$. Its derivative is simply the eigenvalue density $\rho(\lambda)$. Comparison of this observable on quenched configurations $\left(N_{f}=0\right)$ with those in the chirally symmetric phase $\left(N_{f}=56\right)$, see Fig. 3 (left), yields qualitative differences of the spectra in the infrared: The $N_{f}=0$ curve starts linearly from the origin, reflecting an eigenvalue density approximately constant near $\lambda=0$. On the contrary, $v(\lambda)$ for $N_{f}=56$ shows a spectral gap for small eigenvalues, implying $\rho(0)=0$, which is consistent with chiral symmetry restoration according to the Banks-Casher relation. The crucial question is on which scale does this spectral gap depend. To answer this question, in Fig. 3 (right) we compare $v(\lambda)$ at $N_{f}=56$ for various lattice volumes, $L=4,6,8,10$ and 12 . We find that the spectral gap scales $\propto 1 / L$ to a good approximation, which is a strong indication that our theory is IR-conformal ${ }^{7}$ : There does not seem to be any length scale in the chirally restored phase other than $L .^{8}$

maximally disordered): the ordering effect of the dynamical fermions plays a dominant role, and the vanishing of the plaquette coupling is not associated with special properties.

${ }^{6}$ The extracted value of $g(L)$ approaches $g^{*} \sim 0.95$ and $\sim 0.80$ for $N_{f}=56$ and 96 respectively. So $g^{*}$ decreases as $N_{f}$ increases: as $N_{f}$ keeps increasing, the ordering effect of the fermions increases, all Wilson loops are driven towards 1 . The theory becomes trivial for $N_{f} \rightarrow \infty$, even in the strong-coupling limit. We come back to this point in Sec. 4 .

${ }^{7}$ If one would take the limits $L \rightarrow \infty$ first, then $m_{q} \rightarrow 0$, the expected spectral density for a conformal theory would be $\rho(\lambda) \sim \lambda^{\left(3-\gamma^{*}\right) /\left(1+\gamma^{*}\right)}$. Here, we take the opposite order of limits.

${ }^{8}$ Small deviations from $1 / L$ scaling allow us to extract the anomalous mass dimension $\gamma^{*}$. See [13]. 

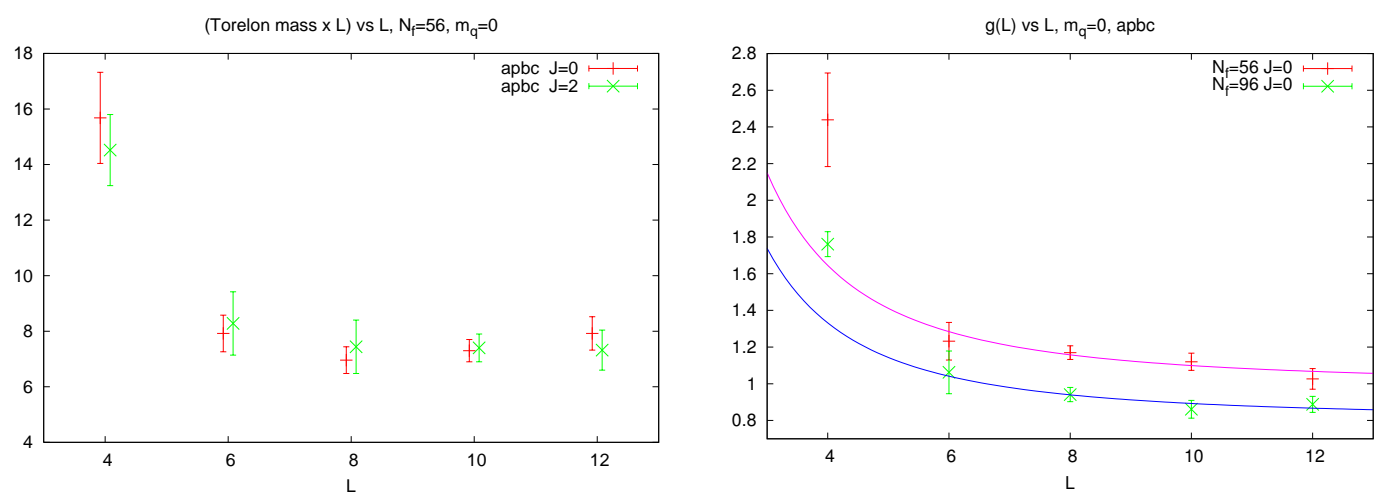

Figure 2: Left: The torelon mass $m_{T}(L)$ multiplied by $L$ versus $L$ for $N_{f}=56$ (for anti-periodic b. c.), which is constant for large enough $L$. Right: The running coupling $g(L)$ defined via the temperature-dependence of the Debye mass, which is identified with the torelon mass, for $N_{f}=56$ and 96 . For each $N_{f}$, the curve is a fit to a constant plus $(a / L)^{2}$ corrections of the 4 largest volumes. The larger $N_{f}$ has a smaller coupling.
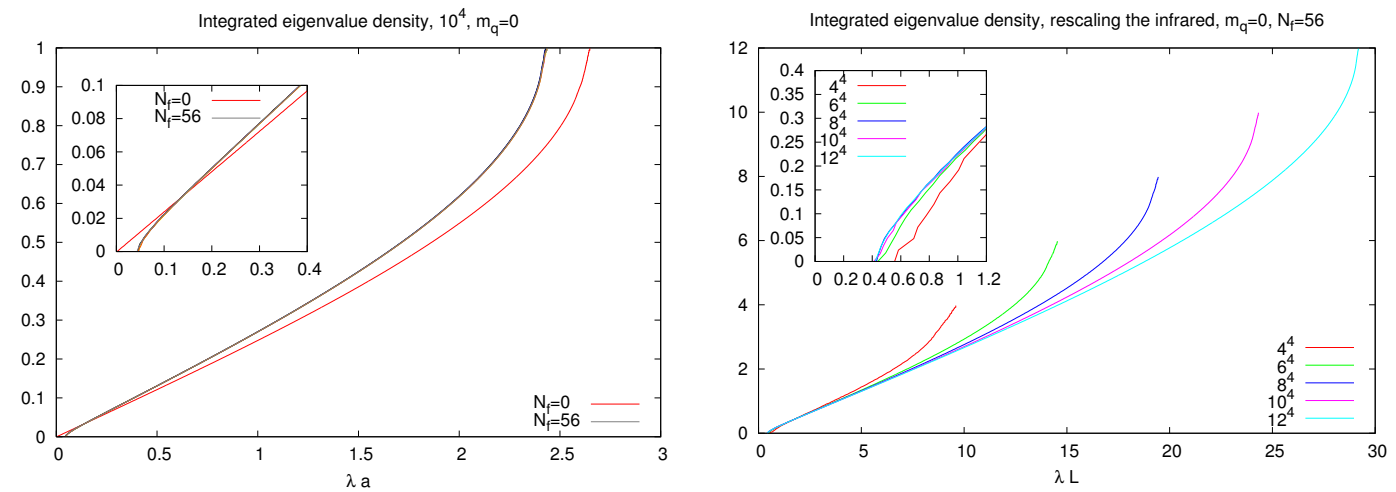

Figure 3: The integrated eigenvalue density. Left: comparison of $N_{f}=0$ (quenched) with $N_{f}=56$ in the chirally restored phase, where only the latter shows a spectral gap. Right: The rescaled spectral gap, indicating $1 / L$ scaling. 10 configurations are superimposed, variations in the spectrum are very small.

\section{The conjectured phase diagram}

We have presented evidence that the chirally restored phase at strong coupling is IR-conformal and non-trivial. In order to make the connection to the conformal window in the continuum, we now want to propose the phase diagram sketched in Fig. 4 (left), as a function of the plaquette coupling $\beta=6 / g_{0}^{2}$ and of the number of fundamental flavors $N_{f}$. Comparing our phase diagram with, e.g., those of Ref. $[8,3]$, one can see substantial differences: in our phase diagram, the $\beta=0$ IR-conformal phase is analytically connected with the weak-coupling, continuum IR-conformal phase - the simplest scenario supported by our exploratory scan in $\beta$ as shown Fig. 1 (right). A single first order phase transition line $N_{f}^{c}(\beta)$ separates the region of broken chiral symmetry at small $N_{f}$ from the chirally symmetric region at large $N_{f}$. Furthermore, based on our results for the running coupling Fig. 2, we conjecture that for any finite $\beta$ and $N_{f}>N_{f}^{c}(\beta)$, large- $N_{f}$ lattice QCD is IR-conformal, with a non-trivial fixed-point coupling $g^{*}>0$. This value changes continuously with $\left(\beta, N_{f}\right)$, reaching the value zero for $\beta=\infty, N_{f}>33 / 2$ and for $N_{f}=\infty \forall \beta$, as indicated Fig. 4 (left) by a thick dotted line. $g^{*}$ grows as one moves away from this dotted boundary towards the phase transition line. An alternative scenario would be that the running coupling in Fig. 2 slowly approaches zero, and $g^{*}=0$ for $N_{f}>33 / 2$ for any $\beta$ in the chirally symmetric phase. This is sketched in Fig. 4 (right). In this scenario, the anomalous mass dimension at $\beta=0$ should vanish, $\gamma^{*}=0$, in contrast to our findings reported in [13]. Finally, one may consider the dashed line $g^{*}=g_{0}$, with $g_{0}=\left(2 N_{c} / \beta\right)^{1 / 2}$, where the IR fixed-point coupling has the same value as the bare coupling. This 

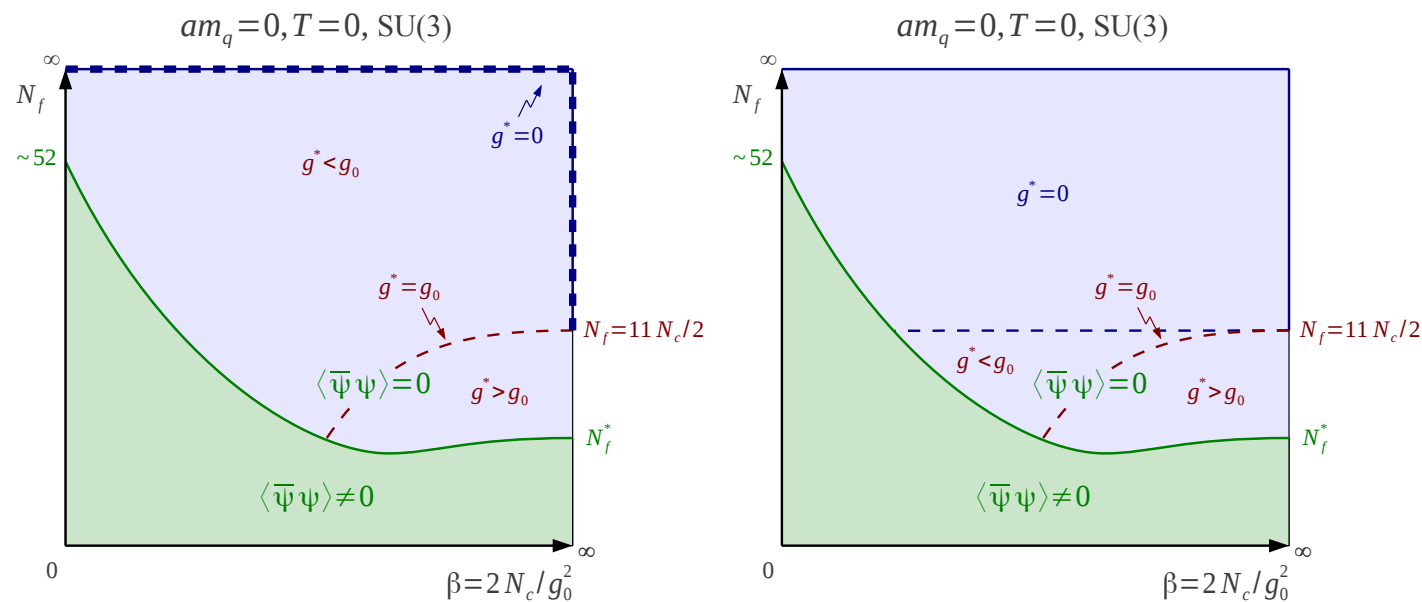

Figure 4: Left: conjectured phase diagram in the $\left(\beta, N_{f}\right)$ plane. A single phase transition line separates the chirally broken phase from the chirally symmetric, IR-conformal phase. The thick dotted line indicates trivial theories. Right: alternative scenario, where the trivial theories extend to the area above $N_{f}=11 N_{c} / 2$. It is not favored by our measurements.

line starts at the point $\left(\beta=\infty, N_{f}=33 / 2\right)$ where $g^{*}=g_{0}=0$. Its precise location depends on the chosen renormalization scheme. It is not associated with any kind of singularity of the free energy.

We have determined the phase diagram in the strong-coupling region only. Studying the continuum limit is of course much more difficult, due to the large lattices that have to be used in order to control the finite size effects and the difficult control of lattice artifacts. We would like to suggest that the strongcoupling limit may represent an advantageous "poor man's laboratory" for the study of $4 d$ IR-conformal gauge theories: the range of scales over which conformal invariance applies for a given computing effort is greatly enhanced at strong coupling. ${ }^{9}$

\section{Conclusion}

We have shown that for $\beta=0$, contrary to common wisdom, there exists a strongly first-order, $N_{f}$-driven bulk transition to a chirally symmetric phase. In the chiral limit, the transition occurs for $N_{f}{ }^{c}=52(4)$ continuum flavors. This finding is in contrast to the mean-field prediction, whose failure can be traced back to approximations relying on $N_{f}$ being small. Clearly, the conventional, automatic association of the strong-coupling limit with confinement and chiral symmetry breaking is too naive. Furthermore, the chirally restored phase extends to weak coupling.

We have also shown numerical evidence that the $\beta=0$ chirally restored phase of "large- $N_{f}$ QCD" is IR-conformal, with a non-trivial, $N_{f}$-dependent value of the IR fixed-point coupling. Since we have not observed any evidence for an additional $T=0$ phase transition as $\beta$ is increased, we speculate that the strong coupling chirally symmetric, IR-conformal phase is analytically connected with the continuum IR-conformal phase.

One may ask how robust these statements are with respect to the particular discretization of the Dirac operator and the gauge action. We think that the qualitative features will remain: chiral symmetry breaking at strong coupling is a general consequence of the disorder in the gauge field. The ordering

${ }^{9}$ At weak coupling, for a given lattice size $N^{4}$, the scales are ordered $a \ll 1 / \Lambda \ll L=N a$, where $\Lambda$ is the scale generated by the asymptotically free gauge dynamics. In contrast, at strong coupling, where the lattice becomes maximally coarse, the hierarchy is $a \sim 1 / \Lambda \ll L=N a$. Hence the dynamical range of conformal invariance, characterized by the product $L \Lambda$, is maximized at $\beta=0$ for a given lattice size $N=L / a$. 
effect of fermions also is generic. So we do expect an $N_{f}$-driven bulk transition, generically of firstorder. Actually, such a transition was observed for Wilson fermions in Ref. [9]. A first-order transition to a chirally broken phase as $\beta$ is reduced at fixed $N_{f}$ has been observed many times for various lattice actions [10]. If the first-order nature persists all the way to the continuum limit, then walking dynamics will not be observed, and the transition to the conformal window will be characterized by "jumping dynamics", as proposed by Sannino [11].

There are many directions in which to extend this exploratory study. More observables, like the static potential, should be studied. ${ }^{10}$ One could study the $\left(\beta, N_{f}\right)$ phase diagram as the gauge group or the fermion content is changed. Also, and to make contact with other numerical studies, a mass deformation can be introduced. ${ }^{11}$

\section{Acknowledgements}

We are grateful for stimulating discussions with P. Damgaard, L. Del Debbio, A. Hasenfratz, A. Kovner, A. Patella, K. Rummukainen, F. Sannino, K. Splittorff, B. Svetitsky and R. Zwicky. Computations have been carried out on the Brutus cluster at the ETH Zürich and on a small cluster in the Sejong University physics department. S. K. is supported by Korea Foundation for International Cooperation of Science \& Technology (KICOS). W. U. is supported by the Swiss National Science Foundation under grant 200020-122117.

\section{References}

[1] For a review, see L. Del Debbio, arXiv:1102.4066 [hep-lat].

[2] P. de Forcrand, M. Pepe and U. -J. Wiese, arXiv:1204.4913 [hep-lat].

[3] V. A. Miransky and K. Yamawaki, Phys. Rev. D 55 (1997) 5051 [Erratum-ibid. D 56 (1997) 3768] [arXiv:9611142 [hep-th]].

[4] H. Kluberg-Stern, A. Morel and B. Petersson, Nucl. Phys. B 215 (1983) 527.

[5] P. H. Damgaard, D. Hochberg and N. Kawamoto, Phys. Lett. B 158 (1985) 239.

[6] A. Hasenfratz and T. A. DeGrand, Phys. Rev. D 49 (1994) 466 [hep-lat/9304001].

[7] C. Michael, Phys. Lett. B 232 (1989) 247.

[8] T. Banks and A. Zaks, Nucl. Phys. B 196 (1982) 189.

[9] Y. Iwasaki et al., Phys. Rev. Lett. 69 (1992) 21; K. -i. Nagai et al., Phys. Rev. D 80 (2009) 074508 [arXiv:0908.0166 [hep-lat]].

[10] J. B. Kogut and D. K. Sinclair, Nucl. Phys. B 295 (1988) 465; P. H. Damgaard et al., Phys. Lett. B 400 (1997) 169 [hep-lat/9701008]; A. Deuzeman et al., Phys. Rev. D 82 (2010) 074503 [arXiv:0904.4662 [hep-ph]]; X. -Y. Jin and R. D. Mawhinney, PoS LATTICE 2011 (2011) 066 [arXiv:1203.5855 [hep-lat]]; A. Cheng, A. Hasenfratz and D. Schaich, Phys. Rev. D 85 (2012) 094509 [arXiv:1111.2317 [hep-lat]].

[11] F. Sannino, arXiv:1205.4246 [hep-ph].

[12] P. de Forcrand and O. Jahn, Nucl. Phys. B 651 (2003) 125 [arXiv:0211004 [hep-lat]].

[13] S. Kim, Ph. de Forcrand and W. Unger, to appear in JHEP [arXiv:1208.2148 [hep-lat]].

\footnotetext{
${ }^{10}$ We have also studied the hadron spectrum: we find that parity partners are degenerate, and masses are exclusively due to finite-size effects: all hadron masses go to zero as the lattice size $L$ is increased. Moreover, mass ratios such as $m_{\pi} / m_{\rho}$ should become constant. Our results are reported in [13].

${ }^{11}$ We have studied the quark mass dependence of the chiral condensate, but there are many technical difficutlies associated with extracting the anomalous dimension $\gamma^{*}$, which we explain in [13].
} 